УДК 658.512 .2

DOI: $10.15827 / 0236-235 X .125 .081-087$
Дата подачи статьи: 26.06 .18

2019. Т. 32. № 1. С. $081-087$

\title{
Прогнозирование развития технических систем на основе закономерностей их строения при использовании банков фундаментальных знаний
}

\author{
Д.В. Бутенко 1, к.т.н., доиент, gindenburg@mail.ru \\ 1 Волгоградский государственный технический университет, г. Волгоград, 400005, Россия
}

\begin{abstract}
Настоящая статья посвящена применению технологий к инженерно-техническому творчеству как генеральному аттрактору научно-познавательной деятельности. Инженерное творчество как самостоятельная дисциплина опирается на фундаментальные законы строения, функционирования и развития систем, которые имеют первостепенное значение для практики получения новых решений в любой области знания. В статье на примере химии и химической технологии обосновывается необходимость использования указанных законов, что вытекает прежде всего из требований получения практически пригодных технико-технологических решений, демонстрирующих качественно новые состояния проектируемых систем и новых технических изделий. Применение для химии обусловлено наличием формализации представления информации в данной области
\end{abstract} знания.

Активное использование современных компьютерных технологий позволяет эффективно решать задачи базовой проблематики общих законов строения и развития техники - установление и применение изоморфных закономерностей для технических объектов различной природы, которые, в свою очередь, являются объективной основой для открытий, изобретений и совершенствования техники и технологий.

В статье рассмотрены аналогичные современные разработки, показаны их достоинства и недостатки и представлена авторская интеллектуальная технология использования указанных закономерностей в области химической технологии в сочетании с использованием инструментария автоматизированных банков фундаментальных знаний. Описанные результаты открывают общие перспективы технологий технического творчества - возможность получения патентоспособных результатов интеллектуальной деятельности с высокой степенью новизны и конкурентоспособностью, которые могут быть усилены с помощью новейших вычислительных средств.

Ключевые слова: инженерное творчество, техническое творчество, закономерности развития систем, закономерности строения систем, банки фундаментальных знаний, банк химических реакиий «Орхидея».

Способность к творчеству - целевая человеческая функция, благодаря которой человек может считать себя личностью, активным творцом настоящего и будущего. Эта способность открывает субъекту возможность реализовать свой внутренний потенциал и дает ощущение значимости в социуме. Технологии инженерного технического творчества, в свою очередь, открывают путь к настоящему свободному развитию личности. Инженерное творчество опирается на общесистемные закономерности строения и развития систем, является синтезом технических, творческих и психологических знаний и может стать самостоятельным научным направлением в новом социально-технологическом укладе. В последние годы оно стало предметом пристального изучения, применяется в различных областях науки, техники и технологий и используется для перехода к прорывным решениям и технологиям. Сейчас в России внедрение технологий инженерного творчества - единственное объективное обеспечение технологического и социального прорыва и получения экономики нового типа, экономики знаний, способной создать условия не только для выживания, но и для достойных нравственных, экономических и технологических достижений социума.

Закономерности строения, функционирования и развития технических систем (ТС) являются фундаментом любого исследования и имеют первостепенное значение при проектировании новых ТС. Разработка новых веществ и технических материалов строится не только на знаниях о существующих веществах, материалах и особенностях их применения, но и на закономерностях строения и развития техники в целом. Под закономерностями для ТС в концептуальном проектировании понимаются суждения, которые отражают всеобщую, существенную, устойчивую и воспроизводимую зависимость между множествами признаков и свойств элементов и составных частей ТС [1].

В современной химии разработка новых веществ и материалов, которые допустимо рассматривать как системы, происходит тремя возможными путями: 1 параметрическая оптимизация количественных соотношений компонентов при неизменной структуре; 2 создание нового технического решения при выборе новой структуры; 3 - выбор нового принципа создания материала, нового принципа действия. При выполнении поисковых процедур на цикле 1-3 происходит рост критериев эффективности при росте затрат на НИР в соответствии с S-функцией [2], которая описывает тенденции развития не только отдельных типов веществ и материалов, но и всего класса материалов одного функционального назначения. S-функция может быть понята как общесистемная закономерность развития, она наглядно показывает четко детерминированную стадийность: в то время как одни системы находятся на стадии проработки, а другие - активного роста, третьи переходят к затуханию. Одновременное 
с развитием выполнение системами многих функций, ужесточение условий эксплуатации и увеличение разнообразия эксплуатационных воздействий приводят к сложности технических объектов [3]. Усложнение каждого класса систем происходит, прежде всего, за счет увеличения числа физико-химических эффектов, что невозможно отследить без использования современных возможностей вычислительной техники и банков фундаментальных знаний о веществах, материалах, протекании процессов их эксплуатации и способах их создания.

Еще одним наиболее изученным с количественной точки зрения закономерным явлением в развитии ТС является изменение системы в пределах существующего приниипа действия (ПД). Здесь наиболее интересным представляется решение вопроса о том, какой должна быть система, приходящая на замену старой, то есть решение задачи технической прогностики. Так, продвижение системы по кривой S-функции хорошо описывается установленными закономерностями развития ТС $[4,5]$.

Анализ известных закономерностей развития показывает, что они могут быть описаны в терминах изменения параметров ТС на различных системных уровнях в сторону экстремальных значений. В своем развитии $\mathrm{TC}$ передвигается по $\mathrm{S}$-образной кривой и стремится пережить на ней экстремум.

Наиболее ярко требования к достижению экстремума раскрываются в следующих известных закономерностях строения и развития систем:

- закон соответствия между функцией и структурой;

- закономерность оптимального соотношения параметров;

- закономерность минимизации компоновочных затрат;

- закон стадийного развития техники;

- закономерность функционального развертывания системы;

- закономерность повышения динамичности и управляемости системы;

- закономерность дифференциации и специализации техники.

В связи с этим представляется актуальным рассмотрение вариантов проектируемой системы, когда один из ее параметров или группу параметров (решающая фактор-группа) можно искусственно установить в экстремальное значение соответственно одной или нескольким закономерностям развития. В связи с тем, что любая система имеет сложное строение в качественном и количественном планах, применение компьютерной техники становится обязательным условием при проектировании нового.

Применение описанных закономерностей для области теоретических основ химии, химической технологии, кибернетики химико-технологических процессов, системотехники химических производств с при- менением возможностей современных ЭВМ открывает качественно новые перспективы получения патентоспособных решений.

Сегодня интенсивно решаются задачи создания автоматизированных банков данных и банков знаний, обеспечивающих высокий уровень принятия решений в органической химии, при анализе и синтезе прогрессивных химико-технологических систем (ХТС) [6].

Для получения новых рациональных семантических решений необходимо переработать большое количество разнообразных знаний, причем эти процедуры, как правило, не связаны с вычислительными операциями. В этой части применение компьютерных технологий необходимо для быстрого и эффективного формирования поискового поля для инициации ассоциативного синтеза технических решений, а также для выбора эффективных эвристик для поддержки генераций новых творческих решений.

В последнее время развитию, усложнению и структурированию химических знаний в органической химии во многом способствовало взаимодействие с вычислительной техникой. Достаточно сказать, что первые работы в области искусственного интеллекта, в частности, известный проект «Эвристический Дендрал», были выполнены в химии [3]. Это стало возможным благодаря высокой формализации знаний в области химического синтеза. Привлекающим фактором для применения вычислительной техники является то, что проблема представления знаний в современной химии во многом решена. Многие технологические схемы химических производств и синтезы органических соединений представлены в виде цепочек химических и физико-химических превращений.

Использование вычислительной техники дает возможность не только запоминать сведения об отдельных химических взаимодействиях (химическая реферативная служба США Chemical Abstracts peферирует ежегодно до 500000 статей), но и вести электронные банки данных по синтетическим реакциям, например, программу CDRS, международный банк ORAC. Эти банки содержат от 15000 до 55000 описаний химических реакций [3]. Наличие большого количества информации по конкретным химическим превращениям, естественно, провоцировало химиков на создание компьютерных систем, самостоятельно строящих «деревья синтеза» - маршруты проведения реакций и анализирующих возможность проведения реакций тем или иным способом [7].

Для эффективного освещения целей настоящей работы необходимо охарактеризовать состояние работ в области компьютерной поддержки органического синтеза, химической технологии и концептуального проектирования. Следует также отметить, что существуют две задачи планирования синтеза: прямая, когда надо осуществить целевое превращение исходного продукта в конечные или найти альтернативные пути реакций преобразования исходного соединения, и об- 
ратная, когда необходимо найти цепь предшественников и их трансформаций, ведущих к получению целевого продукта. Первое направление называется синтетическим, второе - ретросинтетическим.

Программы компьютерного синтеза по-разному ориентированы на пользователя. «Дерево синтеза» может быть построено при участии пользователя в диалоговом режиме или автоматически. Наиболее интересными для данной работы оказались системы Lhasa, SynChem, Mycrosynthese, React, CHIRP [8, 9].

Lhasa и SynChem - наиболее развитые программы с большими библиотеками трансформаций, они содержат набор тестов, определяющих принципиальную возможность проведения той или иной реакции. Каждая реакция в программе Lhasa имеет следующее описание:

- название, субструктура (необходимое структурное окружение);

- характер трансформации (тип структурных изменений);

- условия протекания соответствующей реакции;

- пределы применимости и ограничения.

Программа SynChem [10] оперирует набором тестов, представленных в виде логических функций. В результате машиной формируется специальный код, позволяющий оценить трансформацию, приписать ей соответствующий рейтинг или сообщить о невозможности осуществления данного взаимодействия. Как положительный момент необходимо также отметить стремление использовать в качестве базового принципа ту или иную классификацию органических реакций в виде либо изменения типа связи на реакционном центре, либо основных типов реакций: присоединения, замещения, диссоциации, элиминирования, перегруппировки. Аналогичный классический подход реализован в программе GSS.

Программы CHIRP [8] и React [11] применяются для моделирования процессов в химической технологии. Данные по системе React практически отсутствуют, а относительно системы CHIRP известно, что она использует ограниченный набор реакций, таких как галогенирование, гидрирование, дегидрогалогенирование, дегидратация и дегидрирование, для оптимизации процессов промышленного органического синтеза. Программа может подбирать последовательность реакций.

Известна английская развивающаяся система базы знаний в области органической химии ChEBI $[9,11]$, созданная на языке Јava в Оксфорде в виде открытого веб-приложения БД для сети Интернет. ChEBI - это БД и онтология химических веществ, представляющих интерес для конструирования биологически активных веществ. Она включает в себя более 46000 описаний, каждое из которых классифицируется в рамках онтологии и имеет несколько аннотаций, включая химическую структуру, перекрестные ссылки на БД, синонимы и ссылки на литературу.
Из программ, созданных в России, следует отметить Фламинго (МГУ) [6], в которой реализован формально-логический подход к синтезу химических структур. Программа в основном предназначена для рассмотрения путей синтеза каркасных соединений. В настоящее время она не имеет сопряжения с какойлибо эмпирической БД, что сильно ограничивает ее применение.

В Новосибирском государственном университете создана обучающая программа ДИСФОР, основным недостатком которой с точки зрения целей настоящей работы является неприменимость для планирования органического синтеза.

База знаний BiNChE реализует один из наиболее важных инструментов для работы с онтологиями химических биологически активных веществ - категориальный статистический анализ дифференциального обогащения для больших данных, возникающих в результате современных высокопроизводительных измерений при анализе химических соединений $[12,13]$. Это приложение также создано для работы в сети Интернет.

Сейчас в США создан и активно развивается открытый банк данных с интеллектуальными функциями PubChem [14]. Это открытое хранилище для химических структур, биологически активных веществ с биомедицинскими аннотациями. В нем реализованы семантические веб-технологии, которые находят все большее применение в распространении и интеграции научных данных. Предоставление данных PubChem семантическим веб-службам предназначено для помощи в автоматизации и интеграции больших данных в области химии.

Для автоматизации работ по созданию банков знаний в настоящее время за рубежом созданы специальные языки описания знаний [15], облегчающие трудоемкие работы с большими данными и онтологиями. Например, язык веб-онтологии OWL 2 используется для области химии и химических технологий как наиболее структурированной области научного знания и является языком онтологии для семантической сети с формально определенным значением. Онтологии OWL 2 предоставляют классы, свойства, отдельных семантических агентов и значения данных и хранятся в виде особых форматов документов [16].

Краткий обзор литературы позволяет сделать вывод, что направление исследований по созданию компьютерных систем и банков знаний для химии и органического синтеза в настоящее время активно развивается, но пока есть трудности из-за высокой трудоемкости интеллектуальной разработки в части необходимости обобщения на высоком уровне абстракции текущей химической информации и повышенных требований к прагматической направленности. Однако использование уже созданных банков фундаментальных знаний весьма эффективно и позволяет быстро смоделировать сразу несколько линеек технологиче- 
ских процессов, таким образом построив возможную линию развития выбранной ТС, особенно на уровнях функций и функциональных структур (ФС) и ПД.

Для рассмотрения вариантов ФС и ПД были спроектированы и реализованы банк данных по химическим реакциям и банк данных по физическим эффектам БД «ФЭЯ», структурированный по техническим функциям. При проектировании банка данных химических реакций «Орхидея» [17] учитывались современные тенденции инженерного творчества в части использования общесистемных закономерностей строения и развития систем.

Банк зарегистрирован в Роспатенте (свид. о гос. регистр. программы для ЭВМ № 2017619642). Он построен таким образом, что дает возможность не только проводить многоаспектный поиск химических реакций и получаемых веществ, но и через обращение к химическим функциям осуществлять построение цепей химических реакций на уровне функциональной структуры и конкретных химических взаимодействий. Поиск реакций на основе входящих веществ и желаемой структуры производится с помощью формирования запроса к банку реакций (рис. 1), где пользователь выбирает необходимые элементы поискового образа и может просматривать хранящуюся историю запросов с их результативностью. Помимо основной функции поиска реакций, существует возможность синтеза цепей реакций (рис. 2) на основе как входных данных прямой синтез, так и выбора требуемого выходного ве- щества - обратный синтез. Цепь реакций представляет собой концепт конкретной химической технологии и может быть реализована в различной технологической интерпретации и объемах производства.

Таким образом, с помощью современных банков фундаментальных данных на примере области химических процессов и технологий создается новая информационная технология, открывающая возможность получать информационные модели (концепты) технологических процессов путем автоматического построения вариантов ФС в соответствии с какой-либо закономерностью развития ТС. Это, в свою очередь, не только открывает новые возможности для технического творчества, но и позволяет моделировать и на информационном уровне управлять эффективностью синтеза химических веществ и предсказывать создание новых веществ, материалов и технологических процессов их получения.

Например, в качестве примера использования банка фундаментальных знаний рассмотрим построение возможных маршрутов синтеза ремантадина - известного лекарственного препарата для лечения гриппа на ранних стадиях заболевания. Данный выбор обосновывается тем, что описываемая схема реакции является базовой и открывает возможность построения линеек технологических процессов по созданию не только ремантадина, но и его производных - биологически активных химических веществ и соединений. Реализованный в современной промышленности спо-

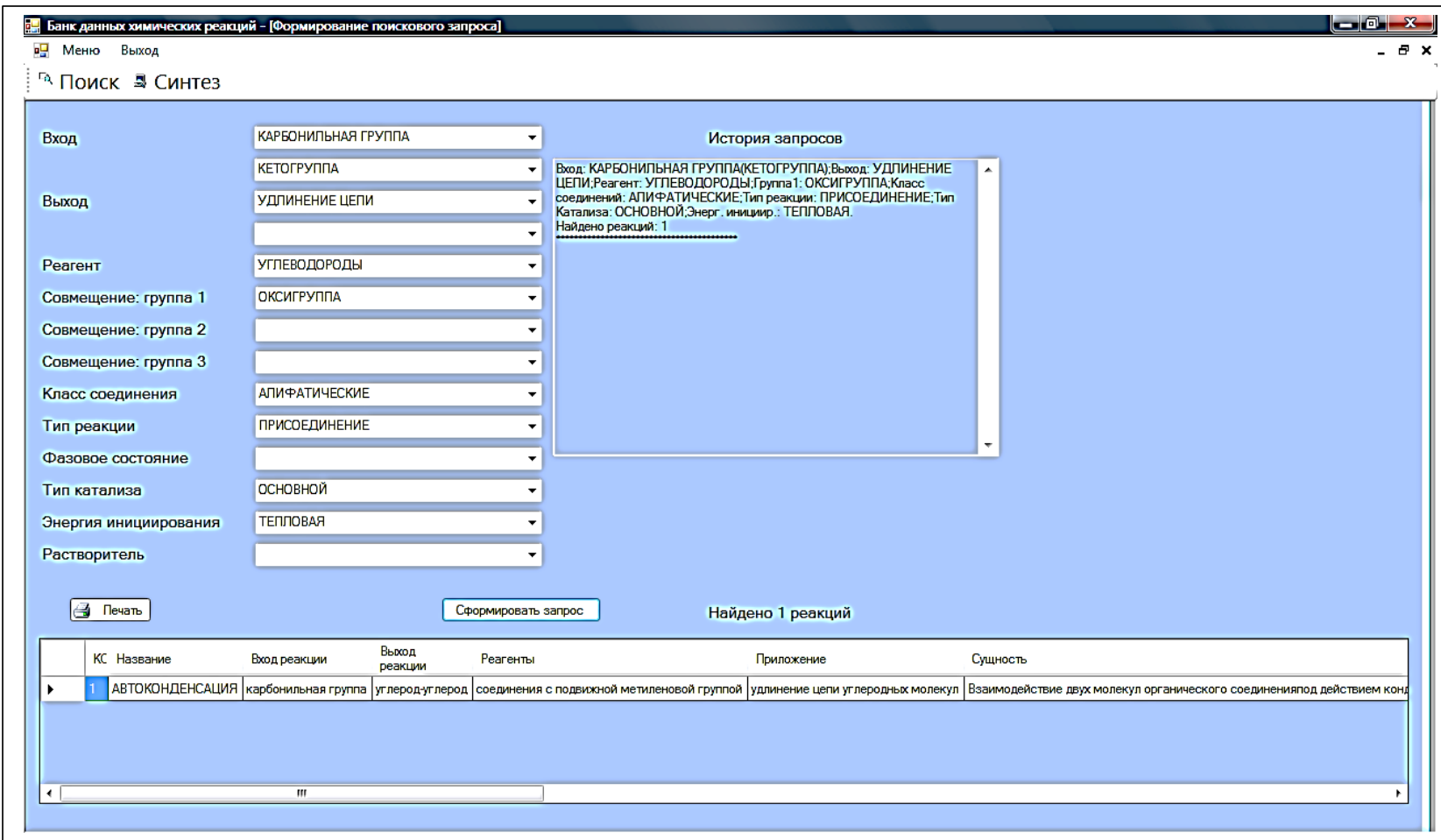

Рис. 1. Формирование поискового запроса к банку химических реакций «Орхидея»

Fig. 1. Formation of a search query to the Orkhideya chemical reaction bank 


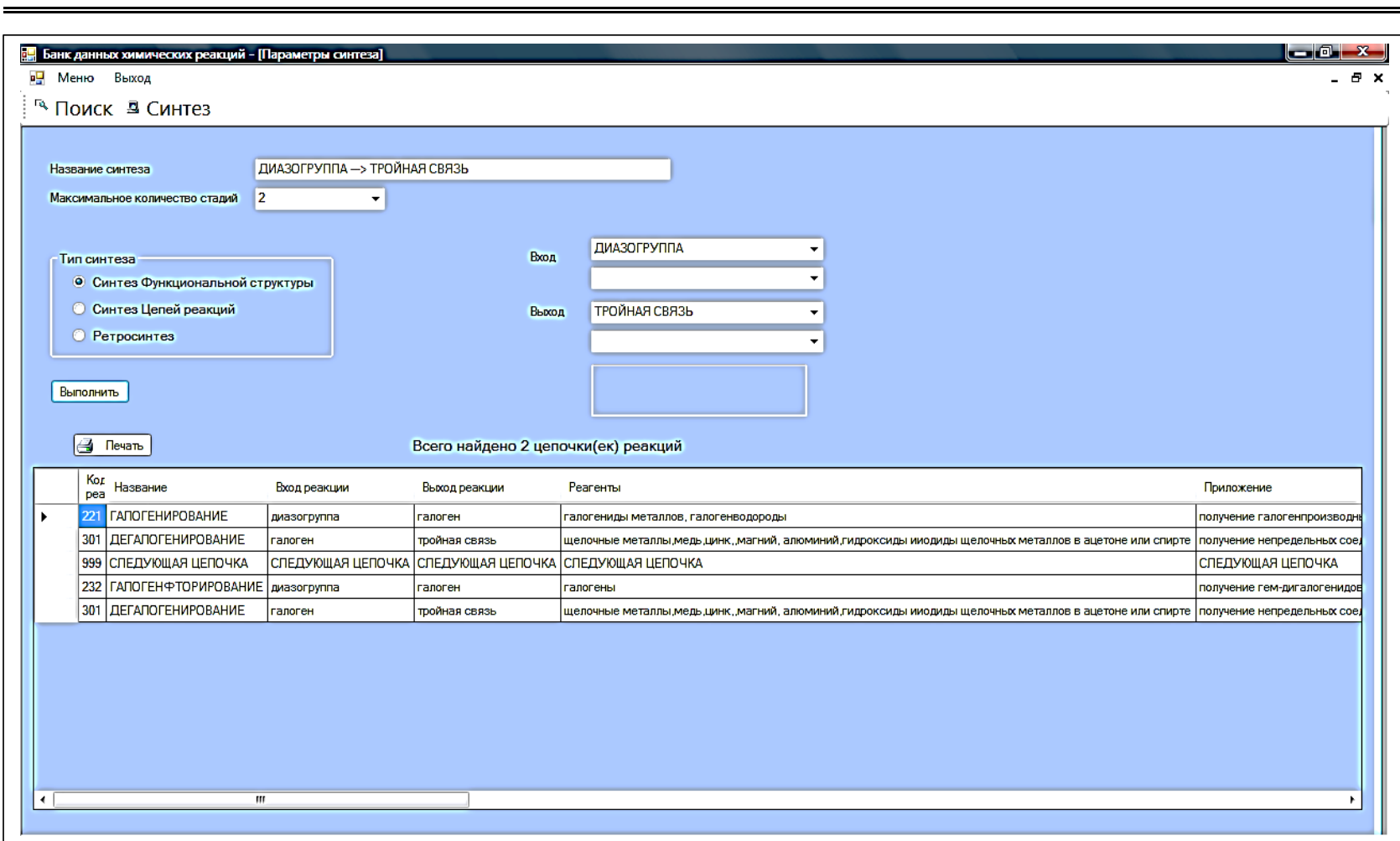

Рис. 2. Возможность синтеза функииональных структур в банке «Орхидея»

Fig. 2. The possibility of the synthesis of functional structures in the Orkhideya bank

соб представляет собой цепь химических взаимодействий адамантана $(\mathrm{Ad})$ :

$\mathrm{Ad}-\mathrm{H} \rightarrow \mathrm{Ad}-\mathrm{Br} \rightarrow \mathrm{Ad}-\mathrm{COOH} \rightarrow \mathrm{Ad}-\mathrm{COCl} \rightarrow$ $\rightarrow \mathrm{Ad}-\mathrm{COCH}_{3} \rightarrow \mathrm{Ad}-\mathrm{CH}\left(\mathrm{NH}_{2}\right) \mathrm{CH}_{3}[18]$.

Как следует из схемы, синтез финального вещества (ремантадина) включает в себя пять стадий с суммарным выходом на исходный адамантан в $32 \%$.

Смоделируем возможность следования некоторым тенденциям развития ТС, а именно: закономерность минимизации компоновочных затрат и закономерность функционального развертывания ТС.

На уровне ФС это будет соответствовать сокращению числа химических превращений в целевой цепи химических реакций.

Автоматический синтез ФС при помощи банка «Орхидея» для целевого превращения «углеродамин» показал, что возможны одно-, двух- и трехстадийные синтезы выходного вещества.

С целью нахождения конкретных реакций получения ремантадина был сформулирован запрос к системе: имеются ли реакции получения аминов непосредственно из углеводородов, то есть в качестве входного реакционного центра был выбран водород, выходом - аминная группа с условием одновременного удлинения цепи. По такому запросу была найдена одна реакция - аминоэтилирование, у нее существует аналог в виде результатов работы японских авторов, где взаимодействие производится с акрилонитрилом.
С точки зрения существенного изменения химического принципа действия эта реакция заслуживает пристального внимания в аспекте проектирования нового технологического процесса, тем более, что на примере аналогов показана ее принципиальная осуществимость.

Следующим запросом к банку был поиск маршрута, исключающего стадию галогенпроизводного, так как получение его затратно и имеет большие технологические трудности и недостатки. По нему системой было найдено 12 комбинаций с использованием стадии карбоксилирования, которая частично описана в литературе [6]. С точки зрения проектирования новых технологических процессов интересным является предложение системы использовать другие источники СО вместо применяемой муравьиной кислоты, а также винилиденхлорид для карбоксилирования, причем система учла, что получение винилиденхлорида удлиняет функциональную цепочку. Последующий анализ показал, что рассмотрение построения найденной модели технологического процесса с такой точки зрения в литературе отсутствует.

Третьим запросом к банку был поиск маршрута, исключающего стадию карбоксилирования с прямым введением кетогруппы с последующим аминированием. В данном случае системой банка были найдены и предложены к просмотру не описанные в литературе для адамантана реакции галогенацилирования. 
Описанные запросы к банку фундаментальных знаний по химическим реакциям показывают, что химический процесс конструктивно может быть оформлен по-разному. В случае описанного синтеза ремантадина один из основных недостатков - гетерогенность реакции, в которой лимитирующей стадией процесса является смешение твердой и жидкой фаз вещества. В большинстве случаев смешение производится с помощью лопастных или пропеллерных мешалок. Запрос к банку данных физических эффектов (ФЭ) [17], обеспечивающих смешение и интенсификацию химических превращений, позволил отыскать метод ультразвуковой кавитации. Этот ФЭ дает возможность перейти к более прогрессивному ПД, отказавшись от механических макроперемешивающих устройств, что также соответствует закономерности перехода к ретикулярной структуре ТС. Этот пример примечателен тем, что демонстрирует «срастание» химической и физической подсистем процесса.

Таким образом, в статье показано, что использование банков фундаментальных знаний для задач технической прогностики позволяет наглядно представлять креативное поле знаний, оперативно моделировать концепты технологий и производственных процессов, открывающих новые горизонты для технологического прорыва и информационной и инновационной безопасности страны [8]. Но главное - показать подходы к использованию общей системной закономерности появления новых продуктов и систем, возможности управляемого перехода к другим, новым принципам действия, появлению новых технологий, отвечающих закономерностям развития систем. Конкретно для области химии и химических технологий это комплексные химические реакции, которые позволяют в одном технологическом акте совместить выполнение нескольких химических функций, что соответствует тенденции компактизации ФС, а в случае ФЭ - образование комплексных ФЭ, способствующих выполнению сразу нескольких технологических функций.

С системных позиций вышеизложенное позволяет утверждать, что качественный переход системы на другой ПД может быть смоделирован при изменении любого ее параметра, описывающего ФС и ПД на противоположный [19], например, нахождение ФЭ и химической реакции позволяет осуществить технологический процесс не в жидкой, а твердой или газообразной фазе. А это уже совершенно новый подход и новые технологии, требующие исследования, поскольку при получении такой модели возникает задача экспертного оценивания найденного концептуального решения по выбранным критериям эффективности, так как новая модель может проявить конфликты, что приводит к неоднозначности толкования понятия «прогресс» в развитии ТС $[17,19]$.

В целом, несомненно, в новом технологическом укладе центральными когнитивными элементами, открывающими проблему новизны при исследовании и проектировании ТС, будут являться банки фундаментальных знаний с реализацией различных интеллектуальных функций, которые создаются с помощью технологий инженерного творчества. В данном случае такими банками являются БД «ФЭЯ» и «Орхидея». Они могут использоваться для решения задач на различных системных уровнях в качестве целепорождающих систем, а также для выполнения процедур анализа и синтеза ТC.

\section{Литература}

1. Автоматизация поискового конструирования; [под ред. А.И. Половинкина]. М.: Радио и связь, 1981. 344 с.

2. Фостер Р. Обновление производства: атакующие выигрывают. М.: Прогресс, 1987. 272 с.

3. Дорохов И.Н., Меньшиков В.В. Системный анализ процессов химической технологии. Интеллектуальные системы и инженерное творчество в задачах интенсификации химико-технологических процессов и производств. М.: Наука, 2005. 582 с.

4. Балашов Е.П. Эволюционный синтез систем. М.: Радио и связь, 1985.328 с.

5. Степанова Т.И., Трохин В.Е., Кочетыгов А.Л. Экспертная система по выбору конструкционных материалов в технологии химических реактивов и особо чистых химических веществ // Успехи в химии и химической технологии. 2012. № 1. С. 84-87.

6. Дорохов И.Н., Лебедев А.Г., Калуцков С.А. Компьютерные методы поискового конструирования // Успехи в химии и химической технологии. 2013. № 1. С. 62-69.

7. Косенко Д.В., Воронова Л.И., Воронов В.И. Разработка программного обеспечения для обработки сложноструктурированных данных научного эксперимента // Вестн. Нижневартовского гос. ун-та. 2017. № 3. С. 12-19.

8. Суховей А.Ф. Проблемы обеспечения инновационной безопасности в Российской Федерации // Экономика региона. 2014. № 4. C. 141-151.

9. Clayden J., Geeves N., Warren S. Organic Chemistry. Oxford Univ. Pres., 2012, 1261 p.

10. Hastings J.A., Owen G.A., Dekker A.D. ChEBI in 2016 Improved services and an expanding collection of metabolites. Nucleic Acids Research, 2016, vol. 44, is. D1, pp. D1214-D1219. DOI: https://doi.org/10.1093/nar/gkv1031.

11. Hill D.P., Adams N., Bada M., Batchelor C. et al. Dovetailing biology and chemistry: integrating the Gene Ontology with the ChEBI chemical ontology. BMC Genomics, 2013, vol. 14, pp. 513-521. DOI: 10.1186/1471-2164-14-513.

12. Lamurias A., Ferreira J., Couto F. Improving chemical entity recognition through h-index based semantic similarity. J. Cheminform, 2015, no. 7, vol. 1, pp. 13-20.

13. Moreno P., Beisken S., Harsha B., Muthukrishnan V., Tudose I. et al. BiNChE: a web tool and library for chemical enrichment analysis based on the ChEBI ontology. BMC Bioinformatics, 2015, no. 16, p. 56 DOI: $10.1186 / \mathrm{s} 12859-015-0486-3$.

14. Fu G., Batchelor C., Dumontier M., Hastings J.,Willighagen E,.Bolton E. PubChemRDF: towards the semantic annotation of PubChem compound and substance databases. J. of Cheminformatics, 2015 , vol. 7, no. 1, p. 34. DOI: https://doi.org/10.1186/s13321-015-0084-4.

15. Horridge M., Patel-Schneider P.F. OWL 2 Web ontology language Manchester syntax. 2015. URL: http://www.w3.org/TR/owl2manchester-syntax/ (дата обращения: 20.06.2018).

16. Small Molecule Identifier Database (SMID DB). URL: http://smid-db.org (дата обращения: 20.06.2018).

17. Бутенко Д.В., Привалов О.О., Бутенко Л.Н. Объектно-ориентированный банк технических функций и физических эффектов для проектирования химических технологий // Изв. ВолгГТУ: Концептуальное проектирование в образовании, технике и технологии. 2006. Вып. 2. № 2. С. 89-92.

18. Бутенко Л.Н., Дербишер В.Е., Хардин А.П., Шрейберт А.И. 
Синтез адамантанполимеркарбоновых кислот // Журнал органической химии. 1973. Т. 9. № 4. С. 728-729.

19. Бутенко Д.В., Бугрий Р.С. Разработка программной среды поддержки анализа функциональной структуры систем в аспекте свойства целостности // Программные продукты и системы. 2013. № 4. C. 108-113.

Software \& Systems

Received 26.06.18

DOI: 10.15827/0236-235X.125.081-087

2019, vol. 32, no. 1, pp. 081-087

\section{Forecasting of engineering system development based on their morphology when using fundamental knowledge banks}

D.V. Butenko ${ }^{1}$, Ph.D. (Engineering), Associate Professor, gindenburg@mail.ru

${ }^{1}$ Volgograd State Technical University, Volgograd, 400005, Russian Federation

Abstract. This paper describes the use of technologies in engineering creativity as a general attractor of scientific and educational activities. Engineering creativity as a discipline is based on the fundamental laws of structuring, functioning and development of systems that are of high priority for new solutions in any field of knowledge. The paper proves the need for using these laws on the example of chemistry and chemical technology. This is implied by the requirements of obtaining practically suitable technical and technological solutions that demonstrate completely new states of the designed systems and technical products. The formalization of information representation in this field of knowledge determines its application in chemistry.

The active use of modern computer technologies allows effective solving of basic problems of general laws of technology structure and development. They include the establishment and application of isomorphic regularities for different technical objects, which in turn are an objective basis for discoveries, inventions and technology improvement.

The paper considers similar modern developments, shows their advantages and disadvantages and presents the author's intellectual technology of using these regularities in chemical technology in combination with the tools of fundamental knowledge automated banks. The described results show general prospects of technical creativity technologies. It is a possibility of obtaining patentable results of intellectual activity with a high degree of novelty and competitiveness, which might be increased through modern computing.

Keywords: engineering creativity, technical creativity, system development regularities, system structure regularities, banks of fundamental knowledge, Orkhideya chemical reactions bank.

\section{References}

1. Automation of Search Design. A.I. Polovinkin (Ed.). Moscow, Radio i svyaz Publ., 1981, 344 p.

2. Foster R. Innovation. The Attacker's Advantage. Summit Books Publ., 1986, 316 p. (Russ. ed.: Moscow, Progress Publ., 1987, 272 p.).

3. Dorokhov I.N., Menshikov V.V. System Analysis of Chemical Technology Processes. Intellectual Systems and Engineering Creativity in Intensification Problems of Chemical-Technological Processes. Moscow, Nauka Publ., 2005, 582 p.

4. Balashov E.P. Evolution System Synthesis. Moscow, Radio i svyaz Publ., 1985, 328 p.

5. Stepanova T.I., Trokhin V.E., Kochetygov A.L. An expert system for selecting structural materials in the technology of chemical reagents and high-purity chemicals. J. Adnvances in Chemistry and Chemical Technology. 2012, no. 1, pp. 84-87 (in Russ.).

6. Dorokhov I.N., Lebedev A.G., Kalutskov S.A. Kompyuternyye metody poiskovogo konstruirovaniya. J. Adnvances in Chemistry and Chemical Technology. 2013, no. 1, pp. 62-69.

7. Kosenko D.V., Voronova L.I., Voronov V.I. Software development for processing complexly structured data in a scientific experiment. The Bulletin of Nizhnevartovsk State University. 2017, no. 3, pp. 12-19 (in Russ.).

8. Sukhovey A.F. The problems of ensuring innovative security in the Russian Federation. Regional Economy. 2014, no. 4, pp. 141-151 (in Russ.).

9. Clayden J., Geeves N., Warren S. Organic Chemistry. 2nd ed. Oxford Univ. Press, 2012, 1261 p.

10. Hastings J.A., Owen G.A., Dekker A.D. Biocatalysis/Biodegradation Database: ChEBI in 2016 Improved services and an expanding collection of metabolites. Nucleic Acids Research. Univ. of Minnesota Publ., 2016, vol. 44, iss. D1, pp. D1214-D1219. DOI: https://doi.org/10.1093/nar/gkv1031.

11. Hill D.P., Adams N., Bada M., Batchelor C. et al. Dovetailing biology and chemistry: integrating the Gene Ontology with the ChEBI chemical ontology. BMC Genomics. 2013, vol. 14, pp. 513-521. DOI: 10.1186/1471-2164-14-513.

12. Lamurias A., Ferreira J., Couto F. Improving chemical entity recognition through h-index based semantic similarity. $J$. Cheminform. 2015, no. 7, vol. 1, pp. 13-20.

13. Moreno P., Beisken S., Harsha B., Muthukrishnan V., Tudose I. et al. BiNChE: a web tool and library for chemical enrichment analysis based on the ChEBI ontology. BMC Bioinformatics. 2015, no. 16, p. 56. DOI: 10.1186/s12859-015-0486-3.

14. Fu G., Batchelor C., Dumontier M., Hastings J., Willighagen E., Bolton E. PubChemRDF: towards the semantic annotation of PubChem compound and substance databases. J. of Cheminformatics. 2015, vol. 7, no.1, pp. 34. DOI: https://doi.org/10.1186/s13321015-0084-4.

15. Horridge M., Patel-Schneider P.F. OWL 2 Web Ontology Language Manchester syntax 2015. 2017. Available at: http://www.w3.org/TR/owl2-manchester-syntax/ (accessed June 20, 2018).

16. Small Molecule Identifier Database (SMID DB). 2015. Available at: http://smid-db.org (accessed June 20, 2018).

17. Butenko D.V., Privalov O.O., Butenko L.N. Object-oriented bank of technical functions and physical effects to design chemical technologies. Izvestiya VSTU. Conceptual Design in Education, Engineering and Technology. Volgograd, 2006, iss. 2, no. 2, pp. 89-92 (in Russ.).

18. Butenko L.N., Derbisher V.E., Hardin A.P. Synthesis of adamantane polymer carboxylic acids. Russian J. of Organic Chemistry. 1973, no. 9, pp. 728-729 (in Russ.).

19. Butenko D.V., Bugry R.S. Development of software environment to support analysis of the system functional structure in the context of integrity properties. Software \& Systems. 2013, no. 4, pp. 108-113 (in Russ.). 